Einführung zum Thema

Gastroenterologe 2020 15:441-442

https://doi.org/10.1007/s11377-020-00487-y

(c) Springer Medizin Verlag $\mathrm{GmbH}$, ein Teil von Springer Nature 2020

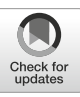

\author{
V. Klippenstein' · S. Ciesek ${ }^{2}$. J. F. Riemann ${ }^{3,4}$ \\ 'Springer Medizin Verlag GmbH, Heidelberg, Deutschland \\ ${ }^{2}$ Institut für Medizinische Virologie, Universitätsklinikum Frankfurt, Frankfurt, Deutschland \\ ${ }^{3}$ Klinik für Gastroenterologie, Infektiologie, Diabetologie und Gastrointestinale Onkologie (Medizinische \\ Klinik C), Klinikum Ludwigshafen, Ludwigshafen, Deutschland \\ ${ }^{4}$ Stiftung LebensBlicke, Ludwigshafen, Deutschland
}

\title{
COVID-19 - eine Multiorganerkrankung stellt das Gesundheitssystem auf die Probe
}

neuartige Virus kann neben den zu Beginn der Pandemie beschriebenen atypischen pulmonalen Komplikationen auch neurologische, nephrologische, kardiologische, gastroenterologische, hämatologische und dermatologische Manifestationen verursachen [1]. Folgen können die Entwicklung von Lungen-, Multiorganversagen sowie eines schweren Inflammationssyndroms sein. Die Komplexität der Erkrankung geht folglich mit besonderen Anforderungen an Infektionsmanagement und Therapieentwicklung einher.

Zum Zeitpunkt des Abfassens dieses Beitrags sind weltweit fast $47 \mathrm{Mio}$. Menschen nachweislich mit SARS-CoV-2 infiziert und insgesamt über 1.200 .000 Tote zu beklagen [2]. Die strikten Hygienemaßnahmen und auch Ausgangsbeschränkungen, die der ersten Infektionswelle im Frühjahr 2020 in Deutschland folgten, resultierten in einem deutlichen Rückgang der Infektionszahlen und lieBen diese in den Sommermonaten auf einem niedrigen und kontrollierbaren $\mathrm{Ni}$ veau halten. Nach wie vor bleibt die Situation jedoch angespannt, die Covid19-Pandemie hat nichts an ihrer Dynamik verloren. Die Zahl der Neuinfektionen steigt seit Ende Juli mit lokalen oder regionalen Ausbrüchen in Deutschland wieder stetig an. Seit Anfang Oktober ist eine rasante Beschleunigung der Übertragungen zu verzeichnen.

Rückblickend auf die letzten Monate lässt sich sagen, dass Virusinfektionen immer noch eine der größten Her- ausforderung für die Medizin darstellen und stetig eine kontinuierliche Neuevaluation und Optimierung der modernen Diagnostik- und Therapiemöglichkeiten erfordern. Dafür ist eine starke interdisziplinäre Zusammenarbeit und ein kontinuierlicher Austausch von wissenschaftlichen Informationen auf überregionaler - ja internationaler Ebene - wesentlich.

In dieser letzten Ausgabe des Jahres haben wir uns vorgenommen, COVID19 aufgrund seiner komplexen Auswirkungen in seiner Gesamtheit als Multiorganerkrankung zu beleuchten. Eingeladen haben wir renommierte Experten aus verschiedenen Fachbereichen, die in den letzten Monaten wesentlich zum medizinischen Verständnis des neuartigen Virus SARS-CoV-2 beigetragen haben. Sie haben grundlegende diagnostische und therapeutische Maßnahmen erarbeitet und die Folgen von COVID-19 auf die medizinische Versorgung hin untersucht.

Unser Schwerpunktheft wird durch einen Beitrag von Salzberger et al. eröffnet. Die Autoren fassen hier die bisher bekannten Parameter und Daten zur Epidemiologie von SARS-CoV-2 zusammen. Dokumentiert werden dabei sowohl epidemiologische Parameter, wie Replikation und Reproduktion des Virus, als auch das Spektrum der Infektionsfolgen unter Betrachtung der Risikofaktoren innerhalb der Population.

Für das Verständnis der epidemiologischen Fragestellungen bezüglich COVID-19 sowie für die Kontrolle des Infektionsgeschehens sind hochsensitive Multisystemerkrankung resultieren. Das 
diagnostische Verfahren unerlässlich. In ihrem Übersichtsartikel gehen Hoehl und Ciesek auf den Erregerdirektnachweis mittels Real-time-Polymerasekettenreaktion (RT-PCR) ein - die Methode der Wahl zur Detektion einer aktiven SARSCoV-2-Infektion - und stellen diesen den Antigen- und Antikörpertests gegenüber. Die Autoren diskutieren die Sensitivität und Spezifität dieser Testverfahren und weisen auf die Bedeutung der Überwachung von Mutationen hin, die die Diagnostik und Therapie beeinträchtigen können.

Auch wenn inzwischen eine Reihe von Organmanifestationen durch SARSCoV-2 beschrieben ist, steht bezüglich des Infektionsverlaufs das Respirationssystem weiterhin im Vordergrund. Pfeifer und Hamer diskutieren die Besonderheiten der COVID-19-Pneumonie aus pathophysiologischer, klinischer und radiologischer Sicht und präsentieren eine Neubewertung der Diagnostik- und Therapieempfehlung für die pulmonale COVID-19-Manifestation.

Neben der Lunge sind vor allem die Nieren ein entscheidendes Zielorgan von SARS-CoV-2. In ihrem Beitrag belegen Rohn et al., dass eine COVID19-Infektion $\mathrm{zu}$ einem akuten Nierenversagen führen kann. Des Weiteren wird die Hochrisikogruppe der Patienten mit chronischen Nierenerkrankungen beleuchtet, die aufgrund ihrer Immundysfunktion sowie der Häufigkeit von Komorbiditäten, wie Diabetes und Bluthochdruck, besonders anfällig für schwere Verläufe einer COVID-19Erkrankung ist.

Durch den weltweit hohen Anteil an intensivpflichtigen Patienten sah sich vor allem die intensivmedizinische Versorgung vor eine große Herausforderung gestellt. Busch et al. informieren eindrucksvoll über die intensivmedizinischen Versorgungsmöglichkeiten und das Abwägen von Interventionen bei Patienten mit schweren COVID-19-Verläufen in der Praxis mit dem Ziel, eine optimale Patientenversorgung zu ermöglichen.

Die COVID-19-bedingte Krisensituation zwang auch die Viszeralmedizin zu umfassenden Anpassungsprozessen, die in kürzester Zeit erfolgen mussten. In ihrem Erfahrungsbericht schildern die Au- toren Banysch et al., wie sich ein Krankenhaus der Grund- und Regelversorgung am Niederrhein der Herausforderung annahm und Versorgungsstrukturen etablierte, um den Schutz der COVID-19infizierten Patienten sowie der Mitarbeiter zu gewährleisten.

Das Unbehagen und die Ängste auf Patientenseite einerseits, die Kapazitätsengpässe sowie vorsorglich verschobene Eingriffe in den Kliniken und Praxen andererseits führten gerade während der ersten Welle der Pandemie zu einem signifikanten Rückgang von Arztkontakten. Die Vorsorgeuntersuchungen in der Gastroenterologie waren hierbei besonders stark betroffen. Mit Fokus auf die Darmkrebsvorsorge widmet sich Riemann den Auswirkungen der COVID19-bedingten Unterbrechung der Vorsorgeuntersuchungen und weist auf die Bedeutung des Darmkrebsscreenings hin.

In unserem letzten Beitrag analysiert Frieling die Folgen der Reduktion der stationären Leistungen für die Gastroenterologie in Deutschland. Die nationalen und internationalen Empfehlungen der Gesellschaften zum Arzt-Patienten-Kontakt sowie die erbrachten gastroenterologischen Leistungen werden hier zusammenfassend dargestellt. Mit Blick auf die Zukunft diskutiert der Autor die uns bevorstehenden Änderungen im deutschen Gesundheitswesen.

Wir danken allen Autorinnen und Autoren für die hochqualitativen Beiträge zu dieser bedeutenden Thematik und hoffen, mit der aktuellen Ausgabe von Der Gastroenterologe einen Beitrag zum Erkenntnisgewinn für die tägliche Arbeit in Klinik und Praxis leisten zu können.

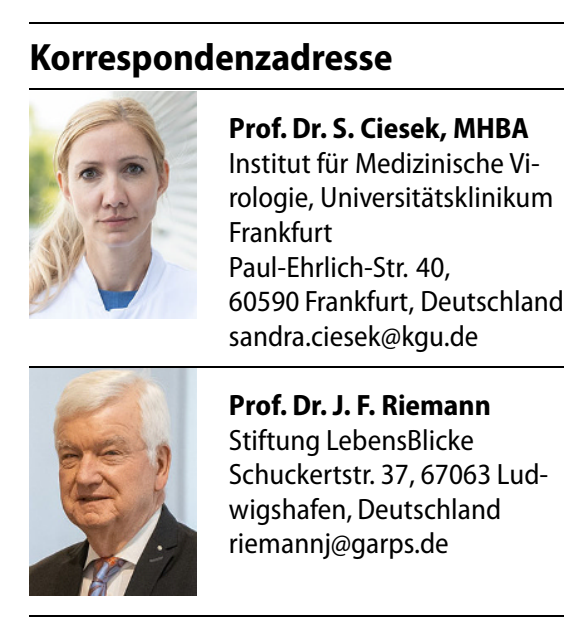

Interessenkonflikt. V. Klippenstein, S. Ciesek und J. F. Riemann geben an, dass kein Interessenkonflikt besteht.

\section{Literatur}

1. Guan W-J et al (2020) Clinical characteristics of Coronavirus disease 2019 in China. N Engl J Med 382:1708-1720. https://doi.org/10.1056/ NEJMoa2002032

2. Johns Hopkins University \& Medicine (2020) COVID-19 dashboard by the Center for Systems Science and Engineering (CSSE) at Johns Hopkins University (JHU). https://coronavirus.jhu.edu/ map.html. Zugegriffen:2. Nov. 2020 